\title{
Evaluación en programas de responsabilidad social corporativa en el sector de la industria química: el caso de la multinacional Quifatex S.A.
}

\author{
Evaluation programs of corporate social responsibility in the sector of the chemical \\ industry: the case of the multinational Quifatex SA
}

\author{
Mónica ARGUELLO \\ Universidad Técnica Particular de Loja (Ecuador) \\ monica.arguello1@yahoo.com \\ Alejandro ÁLVAREZ-NOBELL \\ Universidad Nacional de Córdoba (Argentina) \\ aalvareznobell@eci.unc.edu.ar \\ Recibido: 10/03/2015 \\ Aceptado y Publicado: 10/12/2015
}

\section{Resumen}

A continuación se presentan los resultados de la investigación realizada sobre la gestión de comunicación en la empresa Quifatex, con el objetivo de determinar si cumple o no con cada una de las etapas en el proceso de planificación estratégica en comunicación, especialmente la de medición y evaluación, importantes en la organización para poder identificar herramientas que permitan establecer programas de comunicación con objetivos claros y estratégicos. La metodología utilizada para la investigación fue de tipo exploratorio y descriptivo a través de entrevistas realizadas a los directivos de la organización, así como la técnica de la observación, lo que me permitió estar en contacto directo con el personal de la organización, especialmente con aquellos involucrados en el área de comunicación. Los resultados obtenidos me han permitido concluir que a pesar de que Quifatex es una empresa multinacional con gran trayectoria a nivel nacional e internacional, en el Ecuador tiene todavía mucho camino por recorrer para implementar un programa de comunicación integral que les permita establecer actividades con objetivos estratégicos y que éstos puedan ser auditados y medidos en función de sus resultados. 


\begin{abstract}
The results of the research on the management of communication within the company Quifatex, in order to determine whether it meets each of the stages in the process of strategic planning in communication, especially the measurement and evaluation are presented important in the organization to identify tools to establish clear communication programs and strategic objectives. The methodology used for the research was exploratory and descriptive through interviews with the directors of the organization and observation technique, which allowed me to be in direct contact with the staff of the organization, especially those involved in the communication area. The results have led me to conclude that even though Quifatex is a multinational company with great experience at national and international level, in Ecuador still has a long way to go to implement a comprehensive communications program that allows them to establish activities with strategic objectives and that they can be audited and measured in terms of results.
\end{abstract}

Palabras Clave: Evaluación; responsabilidad social corporativa; comunicación estratégica; Ecuador

Key Words: Evaluation; corporate social responsibility; strategic communication; Ecuador

\title{
1. Introducción
}

Hoy en día las organizaciones afrontan grandes desafíos y vislumbran enormes oportunidades en un mundo globalizado en donde las empresas se ven inmersos en un mercado altamente competitivo, cambiante, por lo cual es indispensable de que las empresas a parte de buscar creatividad para abrir negocios, puedan generar y construir relaciones sólidas con sus clientes internos y externos y por supuesto orientar estas acciones al resultado de la organización.

Una empresa como Quifatex, dedicada a la comercialización, distribución de productos de consumo, farmacéuticos, agrícolas, industrial, entre otros, no es la excepción y ha tenido un marcado crecimiento en la última década, que le exige estar a la vanguardia de estos grandes cambios, especialmente a lo que se refiere a la comunicación integral y estratégica con todas las áreas de la empresa, especialmente con aquellas en donde se genera la imagen corporativa, la cultura empresarial, las buenas relaciones con el entorno, con los stakeholders, empleados, medios de comunicación, comunidad, proveedores, clientes, etc. 
ISSN: $2172-3168$

La base para que la comunicación integral funcione en las organizaciones, es el poder desarrollar planes que aseguren la implantación de estrategias que nos lleven a los objetivos organizacionales, identificar problemas y definir indicadores de gestión. A este escenario donde se define dónde estamos y el qué y cómo hacerlo se denomina "Planificación Estratégica en comunicación", básica para que las organizaciones usen un enfoque más sistemático, lógico y racional para elegir sus estrategias, conocer hacia donde se dirigen y realizar los cambios que sean oportunos.

Tal como lo describe Alvarez (2011:55) "La planificación en la comunicación se convierte así en un escenario teórico-descriptivo que relata todo aquello que hay que hacer y con qué se cuenta para ello. Incluye metas y objetivos, modos o estrategias para conseguir lo que se pretende, tácticas, acciones y herramientas que soporten intenciones y mensajes, etc."

El presente estudio de caso detalla el tratamiento que las organizaciones le dan a la gestión de la comunicación, específicamente a la comunicación estratégica, así como la importancia de la planeación estratégica en comunicación y para que ésta sea integral y funcione, deberá incluir dentro de su proceso, la investigación y evaluación con la finalidad de puedan establecer metas, actividades, objetivos estratégicos y tácticos.

La comunicación organizacional en una empresa como Quifatex, con 1.400 colaboradores, se torna imprescindible y esencial el practicarla integralmente, con el propósito de llegar a tener un alto grado de relacionamiento efectivo, duradero, armónico y transparente con los públicos que cada día demandan estar informados, que exigen el desarrollo de prácticas éticas y socialmente responsables, marcas y productos de calidad e influencia de sus valores e imagen corporativa, identidad y cultura organizacional.

Quifatex ha iniciado el cambio. En el año 2013 con el propósito de tener una comunicación integral, eficiente y guiada por objetivos organizacionales, creó el Área de Comunicación que le permita centralizar tanto la comunicación interna como externa con sus públicos y stakeholders. Como empresa socialmente responsable, Quifatex ha cumplido con sus obligaciones laborales, medioambientales y legales; adicional a la implementación de estrategias de comunicación hacia el campo de la Responsabilidad Social, donde involucró a los colaboradores de la empresa impulsando el reciclaje y el ahorro del papel, para que con los recursos que se obtuviera de esta acción, realizar campañas de responsabilidad con la comunidad. 


\section{Objetivos, Hipótesis y Metodología}

La investigación de este caso se efectuó en Quifatex S.A. de la ciudad de Quito, como matriz en el Ecuador del holding Quicorp. En sus oficinas, ubicadas en la Av. Plaza Lasso y Manuel Zambrano, se obtuvo información a través del Área de Comunicación, Recursos Humanos, Comité de Responsabilidad Social, Área Legal entre otros. Se analizó la gestión de comunicación en la empresa, sus políticas, objetivos, visión, misión, integrantes y el estado actual de la compañía desde enero del 2013 hasta la presente fecha.

La investigación tuvo una duración de cuatro meses, la misma que se inicio cuando se aprobó el tema de la tesis y la empresa asignada para la investigación del caso. Luego del análisis realizado a todos los datos obtenidos de la empresa, se procedió a seleccionar la campaña más importante desarrollada en el año 2013: Responsabilidad Social, donde se analizó la participación activa de los colaboradores con la comunidad. Sobre este caso se llevará a cabo el diagnóstico y la propuesta de mejora, de ser el caso.

La misma tuvo por objetivo general demostrar a través del estudio de caso, durante el año 2013, que la planificación estratégica en comunicación, incluida la función de medición y evaluación de resultados, permiten determinar el aporte de la gestión de comunicación a los objetivos de las organizaciones.

No existieron limitaciones de ninguna naturaleza debido a la vinculación que existe con la compañía, cuyos colaboradores e integrantes de la misma, facilitaron el acceso a la información, recopilación y procesamiento de la misma, con lo que se estuvo en capacidad de desarrollar la investigación sin contratiempos. Sin embargo, el mayor inconveniente presentado fue la reciente creación del área de comunicación, por lo que los datos obtenidos y la planeación estratégica en el estudio de caso, son muy básicos y carecen de un historial, lo que nos permitirá registrar una serie de conclusiones y recomendaciones que le serán útiles a Quifatex para el crecimiento y el buen funcionamiento de un área tan sensible e importante para la compañía.

\subsection{Marco metodológico para el diagnóstico descriptivo}

Quifatex a través de su nueva e integradora Área de Comunicación que nació en el año 2013 y cumpliendo con sus planes y políticas de Responsabilidad Social así como con sus valores corporativos, participó en la Campaña de Responsabilidad Social "Haciendo un mundo mejor" que nació de las actividades de reciclaje y ahorro de papel que se realizaron a nivel de todos los colaboradores de la empresa y permitieron recaudar fondos para ser destinado a dos fundaciones: "Minadores de Sueños" y "Fundación Ecuatoriana Vista para los Ciegos". Al ser una empresa socialmente responsable y con la finalidad de mantener informado a sus stakeholders, colaboradores, proveedores, comunidad en general, Quifatex realizó una campaña de comunicación 
para informar sobre sus actividades de Responsabilidad Social. A través de este estudio de caso, se va a analizar si esta campaña se planificó y gestionó de modo estratégico y si las mismas impactaron a los objetivos organizacionales, para lo cual el marco metodológico nos permitirá recopilar en su fase de investigación descriptiva, el programa de comunicación estratégica que desarrolló Quifatex en el 2013.

Con el fin de dar respuesta al caso estudiado y alcanzar los objetivos propuestos, la presente investigación tendrá un enfoque de tipo exploratorio y descriptivo que nos permitirá identificar los elementos y características del caso de estudio, establecer un análisis de la información recopilada, facilitar la descripción de los hechos y conocer el estado actual de la comunicación en Quifatex.

Es descriptivo, porque según Arias (2004:37) "consiste en la caracterización de un hecho, fenómeno o grupo con el fin de establecer su estructura o comportamiento". De tal manera, este tipo de investigación pretende indagar el comportamiento de las variables comunicación, planificación estratégica, evaluación y objetivos organizacionales. "Los estudios descriptivos buscan especificar las propiedades importantes de personas, grupos, comunidades o cualquier fenómeno que sean sometidos a análisis. Miden o evalúan diversos aspectos, dimensiones o componentes del fenómeno a investigar" (Hernández, Fernández y Baptista, 2005: 20).

\subsection{Técnicas para recolección de datos}

Para Arias (2004), un instrumento de recolección de datos es un dispositivo o formato, que se utiliza para obtener, registrar o almacenar información. Por lo tanto, la aplicación de estas técnicas conducen a la obtención de información, para que sean procesados, analizadas e interpretadas. Las técnicas que se utilizarán para la recolección de datos para medir las variables de estudios, son las siguientes:

\section{- Investigación documental}

Este tipo de investigación tendrá mucha importancia en el estudio del caso ya que para conocer la gestión en comunicación que realiza Quifatex y poder sustentarla teóricamente, se tomó como contribución toda la información relevante y necesaria, tanto la que se indica en textos, como la suministrada por el Área de Comunicación en cuanto a presentaciones institucionales, página Web, guía de procedimientos, artículos, etc.

\section{- Entrevista semiestructurada}

Con el fin de alcanzar los objetivos propuestos, fue necesario emplear esta técnica para captar información de carácter personal por parte de los directivos de la empresa y de las personas que 
tienen relación con la implementación de la gestión de comunicación. En honor al tiempo de los directivos, la entrevista fue enviada vía mail a dos ejecutivos de Quifatex: El Jefe de Comunicación, Paúl Baumgartner y Verónica Reyes, Jefe de Recursos Humanos, quienes procedieron a llenar el cuestionario, cuyo contenido son un banco de preguntas abiertas, basadas en la estructura del "Communication Management Bridge". El objetivo principal es el obtener de los interlocutores, la mayor cantidad de información. La estructura de la entrevista está dirigida para conocer las acciones de comunicación que Quifatex ha desarrollado y la correlación que existe entre la planificación estratégica de la comunicación y los objetivos de la compañía, por lo tanto, basado en la matriz del "Communication Management Bridge", la entrevista incluirá las tres fases integradas para la medición y evaluación: Fase estratégica; Fase Táctica y Fase Operativa; así como las actividades, descripción y aplicación de cada fase.

\subsection{Investigación de campo}

Mediante la observación, se logrará estar en contacto con todo lo concerniente a la campaña de Responsabilidad Social realizada en el año 2013. Por lo tanto, me permite obtener, recolectar y analizar información relevante y actualizada de todos los hechos y acontecimientos que se produjeron en su contexto laboral. De esta manera se podrá alcanzar los objetivos de estudio y plantear una serie de conclusiones y recomendaciones luego de la medición de las variables que intervienen en este estudio de caso. Según Chávez (2003), Población "es el universo de la investigación, sobre el cual se pretende generalizar los resultados. Está constituida por características o estratos que le permiten distinguir los sujetos unos de otros". En el presente estudio de caso la población a ser investigada está conformada por los 4 directivos que conforman el Comité de Responsabilidad Social en Quifatex (Generación Q), de los cuales la muestra está conformada por dos de ellos a quienes se realizaron la entrevista: El Jefe de Comunicación y Publicidad y la Jefe de Recursos Humanos.

\section{Resultados}

A continuación se detalla el informe sobre las variables que nos proporciona el modelo de medición y evaluación en comunicación estratégica, con las respuestas otorgadas por los dos directivos de la empresa a las entrevistas realizadas en forma independiente: Sr. Paúl Baumgartner, Jefe de Comunicación y Publicidad y la Sra. Verónica Reyes, Jefe de Recursos Humanos.

\subsection{Análisis e interpretación fase estratégica}

Al ser Quifatex una organización multinacional con marcado crecimiento en el Ecuador y con apenas un año de creación del área de comunicación, considero que existen grandes oportunidades en la empresa para alinearse a un plan integral de comunicación basado en el "Management Bridge" (proceso de planificación estratégica en comunicación a partir de un modelo para la medición y 
evaluación). Es importante recalcar que actualmente siguen existiendo esfuerzos aislados de las diferentes unidades o ejes estratégicos de la organización, por cuanto no se vinculan o solicitan apoyo a la reciente creada área de comunicación. A pesar de estos procesos aislados, el Área de Comunicación se ha visto en la necesidad de iniciar sus actividades con un programa de comunicación muy sensible y que está en auge dentro de las grandes organizaciones a nivel mundial: La Responsabilidad Social Corporativa. Por esta razón y con la finalidad de definir que los objetivos de comunicación se ajusten a los de la compañía, se realizó previamente una autoevaluación sobre la Responsabilidad Social Corporativa, en la que participaron personas claves en las diferentes áreas de la empresa, lo que le permitió conocer más a fondo las oportunidades y debilidades que la compañía tiene referente a este campo. Con esta autoevaluación, el personal del área de comunicación pudo conocer que Quifatex ha pesar de formar parte de su holding Quicorp, donde ya existen campañas de Responsabilidad Social en los otros países afiliados, no ha realizado ninguna campaña de comunicación referente a este tema en el Ecuador.

La encuesta arrojó un total de puntos de 0,95 , por lo que se alinea a la opción 2: "de $0.81-1.19$ " cuyo diagnóstico es el siguiente:

"Su empresa ya realiza acciones en el ámbito de la RSE. Realice un análisis más detallado del Cuestionario verificando en qué temas obtuvo una puntuación más alta y que contribuyó para ese resultado. Busque prácticas positivas de RSE e ideas sobre cómo desarrollar acciones creativas y formas de superar obstáculos. Siga leyendo las secciones que vienen a continuación para encontrar ideas concretas".

En base a estos resultados, el área de comunicación inició sus actividades en el año 2013 con la campaña de comunicación sobre las actividades de Responsabilidad Social: "implementación del reciclaje y ahorro de papel con la finalidad de obtener recursos que le permitan realizar actividades de Responsabilidad Social con la comunidad".

Se puede afirmar que esta campaña aportó a la gestión de comunicación y los objetivos de la compañía, sin embargo los objetivos definidos fueron bastante generales y se enfocaron en que los colaboradores de la organización, se sensibilicen sobre el manejo de los recursos de la compañía. No obstante, existe un largo camino por recorrer para trazar estrategias de comunicación sólidas, que les permitan cumplir con un modelo de planificación estratégica integral, donde se realice la medición y evaluación de esta campaña y las que a futuro emprendan.

\subsection{Análisis e interpretación fase táctica}

Las dos personas entrevistadas coinciden que la campaña de Responsabilidad Social se determinó a partir de las variables propuestas en los objetivos organizacionales. Ésta afirmación se soporta en que los objetivos de la campaña se realizaron en base a los ya existentes en nuestro holding Quicorp 
en el Perú. Sin embargo, la campaña sobre el reciclaje y ahorro de papel fue una iniciativa tomada en nuestro país, considerando que la acción social pasó a ser un componente natural del proceso de creación de valor, integrada en el concepto de empresa socialmente responsable.

Es importante recalcar que a través de los cuadros y gráficos sobre esta campaña de Responsabilidad Social, se puede determinar que los actores directamente involucrados son todo el personal de la organización, quienes se han concientizado en la importancia de cuidar y ahorrar los recursos de la compañía, para que con lo ahorrado por estos conceptos, se pueda entregar a los más necesitados de nuestra comunidad.

No obstante, esta campaña al ser nueva deberá ser mencionada por todos los medios que tiene la compañía, con la finalidad de refrescar y recordar a los involucrados los beneficios que ofrece la organización para su público externo (comunidad) y así se ejecute una claridad conceptual en los involucrados.

Para realizar la medición y observación de las actividades realizadas en esta campaña, el área de comunicación ha determinado efectuar en este año, una memoria de Responsabilidad Social, en la cual se deberán presentar los resultados de esta campaña, con la finalidad de que todos los colaboradores estén informados sobre lo que está realizando la organización.

\subsection{Análisis e interpretación fase operativa}

Al ser un programa relativamente nuevo con el que la empresa inició sus actividades de Responsabilidad Social en el Ecuador, se puede determinar de acuerdo a las respuestas de los dos entrevistados que si existió un diagnóstico previo para poder conocer el estado actual de la empresa en actividades de Responsabilidad Social Corporativa.

A pesar de haber realizado el diagnóstico previo sobre la situación de Quifatex en cuanto a Responsabilidad Social y al tratarse de una actividad nueva para la organización, la empresa tiene grandes oportunidades no solo para establecer procedimientos formales que permitan medir los cambios en la campaña de comunicación y obtener los dispositivos o herramientas de medición necesarias para verificar los resultados alcanzados durante y posterior a la ejecución de este programa, sino procesarlos y analizarlos para plantear indicadores y metas a lograr.

Siendo Quifatex una multinacional de gran prestigio, que se encuentra ubicado en el puesto 75 de las empresas que realizan Responsabilidad Social Corporativa en el Ecuador, tiene todavía por desarrollar algunos planes de acción a futuro que le permita participar activamente en el desarrollo del país y de la sociedad, con un modelo integral de medición y evaluación en comunicación estratégica. 


\section{Conclusiones}

Luego de haber recolectado la información y desarrollado el análisis respectivo, en relación a los objetivos planteados en el presente trabajo, se procedió a emitir las siguientes conclusiones que respondieron al tema planteado "Planificación Estratégica en Comunicación, el cual se orientó a analizar la aplicabilidad de la medición y evaluación en el plan de comunicación.

Para dar respuesta al primer objetivo específico que estuvo dirigido a "realizar una aproximación a la realidad de la empresa asignada", se efectuó el acercamiento a la organización Quifatex S.A. y a la recientemente creada "Área de Comunicación" y se pudo constatar que en el año 2013 Quifatex inició sus actividades con la campaña de comunicación dirigida a Responsabilidad Social, donde realizaron la implementación del reciclaje y ahorro de papel con la finalidad de obtener recursos que le permitan realizar actividades enfocadas a la campaña.

En tal sentido, para dar respuesta al segundo objetivo específico que estuvo dirigido a "Analizar en el caso seleccionado, mediante la aplicación de un modelo de medición y evaluación al programa de comunicación más importante realizado durante el 2013; la contribución de la comunicación a los objetivos de la organización", se confirmó que esta campaña aportó a la gestión de comunicación y los objetivos de la compañía, pero en términos bastante generales donde se enfocaron en que los colaboradores de la organización, se sensibilicen sobre el manejo de los recursos de la compañía.

En los que respecta al tercer objetivo "Aportar mejoras para la planificación estratégica en la organización, con especial énfasis en la función de evaluación" se pudo concluir que al ser un tema bastante nuevo para la compañía, existe un camino lleno de oportunidades por recorrer para trazar estrategias de comunicación sólidas, que les permitan cumplir con un modelo de planificación estratégica integral, donde se realice la medición y evaluación de esta campaña y las que a futuro emprendan.

Si bien es cierto que el área de comunicación realizó un diagnóstico previo sobre la situación en la que estaba en cuanto a Responsabilidad Social, se debió realizar la evaluación con la finalidad de conocer los logros obtenidos con esta campaña, importante para verificar los resultados alcanzados durante y posterior a la ejecución del programa y así obtener indicadores y metas a alcanzar.

Se observó además que existen deficiencias en cuanto a la comunicación con todas las áreas de la empresa ya que todavía algunas de ésas unidades realizan esfuerzos aislados de comunicación y no solicitan apoyo a esta nueva unidad de la compañía: ARCOM.

\section{Bibliografía}

ALEJANDRO ÁLVAREZ NOBELL (2011): Medición y Evaluación en Comunicación. Colección "Estudios en Relaciones Públicas y Comunicación". No. 2 Málaga: Instituto de Investigación en 
Relaciones Públicas (IIRP). pag. 116. ISBN 9788493783716 - ISBN 9788461536931. http://www.revistacomunicar.com/pdf/2011-10-medicion.pdf

ALEJANDRO ÁLVAREZ NOBELL. La evaluación en comunicación. Desarrollo histórico y principales antecedentes. España. Tenerife, España. 2010. Libro. Artículo Completo. Congreso. II Congreso Internacional Latina de Comunicación Social. Sociedad Latina de Comunicación Social (ISBN: 978-84-938428-0-2). http://www.revistalatinacs.org/10slcs/actas 2010/alejandro cordoba.pdf ALEJANDRO ÁLVAREZ NOBELL. Hacia un modelo integral de medición y evaluación en Comunicación Estratégica: supuestos teóricos, empíricos y metodológicos. V Congreso Internacional Latina de Comunicación Social. Sociedad Latina de Comunicación Social Universidad de La Laguna. 3 al 5 diciembre de 2013. (Tenerife, España). (ISBN-13:978-84-1569829-6) http://www.revistalatinacs.org/13SLCS/2013 actas7131 Nobell.pdf

ÁLVAREZ NOBELL, A. (2008). “Media reputación”. Revista DIRCOM. No. 80. Buenos Aires.

BERTALANFFY VON, L. (1986). General Sistem Theory, G. Braziller, New York.

CAPRIOTTI, P. (2008). Branding Corporativo. Fundamentos para la gestión estratégica de la Identidad Corporativa. Claves del valor intangible de las empresas. Madrid: Pirámide. http://www.bidireccional.net/Blog/BrandingCorporativo.pdf

CASTILLO ESPARCIA, A. (2005). Comunicación Organizacional. Clave Aynadamar, Málaga.

CASTILLO ESPARCIA, ANTONIO (2010): INTRODUCCIÓN A LAS RELACIONES PÚBLICAS (Málaga, España). ISBN: 978-84-614-2448-1- http://antoniocastillo.moonfruit.com/

CHIAVENATO, I. (2009). Comportamiento Organizacional. La dinámica del éxito en las organizaciones. México: McGraw Hill.

COLLE, R. (2002). Teoría cognitiva sistémica de la comunicación. Santiago de Chile: San Pablo..

COSTA, J. (2005). "De la comunicación integrada al director de comunicación". Revista Latinoamericana de Comunicación CHASQUI. Centro Internacional de Estudios Superiores de Comunicación para América Latina Quito, Ecuador. Septiembre, número 091.

COSTA, J. (2009). El Dircom hoy: Dirección y Gestión de la Comunicación en la nueva economía. Barcelona: Gráfic. Granollers.

GÓMEZ AGUILAR, M. (2007). La comunicación en las organizaciones para la mejora de la productividad: El uso de medios como fuente informativa en empresas e instituciones andaluzas. Recuperado el 28 de mayo del 2014 de la página electrónica de la Universidad de Málaga, Facultad de Ciencias de la comunicación, Departamento de Periodismo: http://www.biblioteca.uma.es/bbldoc/tesisuma/17672697.pdf

INSTRUCTIVO GENERAL $\quad$ DEL SISTEMA $\quad$ BIBLIOTECARIO UTPL http://procuraduria.utpl.edu.ec/sites/default/files/Instructivo\%20General\%20del\%20Sistema\%20Bibl iotecario.pdf

LANDA RIVERA, R. (2012) Guía Didáctica de Elaboración de Planes de Relaciones Públicas. LojaEcuador UTPL.

LAURA LESTA; ALEJANDRO ALVAREZ NOBELL. Medición de los aportes de la gestión estratégica de comunicación interna a los objetivos de la organización. Revista PALABRA CLAVE. Bogotá: Universidad de La Sabana. 2011 vol. 14 no. 1 p11 -30. ISSN: 0122-8285 http://palabraclave.unisabana.edu.co/idex.php/palabraclave/article/view/1870 
POZO LITE, M. (1997) Cultura empresarial y comunicación interna: Su influencia en la gestión estratégica. Madrid: Fragua.

QUICORP, 2013. Manual de Imagen Corporativa.

QUIFATEX. Página Web oficial. Recuperado el 6 de abril, 2014, de http://www.quifatex.com.

REVISTA ON LINE "EL PAPEL", recuperado el 4 de julio, 2014 de http://revistaelpapel.com/es/2014/06/ecuador-empresas-quicorp-realizan-actividades-de-ayudasocial/

RíOS, J. (2006). Relaciones Públicas: Su administración en las organizaciones. México: Editorial Trillas.

SALÓ, N. (2005) Aprender a comunicarse en las organizaciones. España: Ediciones Paidós.

WILCOX, L., CAMERON, T. y XIFRA J., (2006), Relaciones Públicas Estrategias y tácticas"

XIFRA, J. (2005). Planificación Estratégica de las Relaciones Públicas. Barcelona: Ediciones Paidós lbérica.

YAGUACHE J. Comunicación Organizacional - Guía didáctica p.23

\section{Forma de citar este artículo en bibliografías}

ARGUELLO, M.; ÁLVAREZ-NOBELL, A. (2015): "Evaluación en programas de responsabilidad social corporativa en el sector de la industria química: el caso de la multinacional Quifatex S.A", en Revista PANGEA № 6, Volumen № 2, páginas 83 a 93. Tenerife: Red Académica Iberoamericana de Comunicación. Recuperado el _ de de 2 de: http://www.revistapangea.org

Sociedad Latina de Comunicación Social Facultad de Ciencias Políticas, Sociales y de la Comunicación

Universidad de La Laguna

Avenida César Marique, s/n; Campus de Guajara

38071 La Laguna, Tenerife (Islas Canarias - España) 